\title{
REAL KAEHLER SUBMANIFOLDS AND UNIQUENESS OF THE GAUSS MAP
}

\author{
MARCOS DAJCZER \& DETLEF GROMOLL
}

The main purpose of this paper is to answer the question: To what extent is a euclidean submanifold determined by its Gauss map? More precisely, let $f, \tilde{f}: M^{n} \rightarrow \mathbf{R}^{n+p}$ be two isometric immersions of a connected riemannian manifold whose Gauss maps into the Grassmannian $G_{n p}$ are congruent. When are $f$ and $\tilde{f}$ congruent?

Classical examples of isometric noncongruent deformations with the same Gauss map are the associated families of minimal surfaces in $\mathbf{R}^{3}$. They are a special case of associated families which can be defined for certain real isometric immersions of Kaehler manifolds which we call circular. It will turn out that locally, all isometric immersions with the same Gauss map can be described in terms of circular submanifolds, whereas globally, additional phenomena arise.

In $\S 1$, we discuss circular submanifolds in spaces of constant curvature. This is related to work of Calabi, Lawson, and others on minimal surfaces. $\$ 2$ deals with circular hypersurfaces. More generally, we classify all Kaehler submanifolds of real codimension 1 , which is of independent interest. In the remaining two sections we show that all isometric immersions $M^{n} \rightarrow \mathbf{R}^{n+p}$ with congruent Gauss maps form a compact abelian group, and we compute its structure.

We would like to thank K. Nomizu for suggesting to look at the above question in the light of some of our previous work.

\section{The associated family of a circular immersion}

Let $M^{n}$ be a connected riemannian manifold of dimension $n$, and $f$ : $M^{n} \rightarrow Q_{c}^{n+p}$ an isometric immersion into a complete simply connected space of constant curvature $c$. We will always assume that $f$ is substantial, i.e. $f(M)$

Received July 24, 1984 and, in revised form, June 14, 1985. Research of the first author was partially supported by CNPq, Brazil, and of the second author by NSF Contract MCS $8102758 \mathrm{~A} 02$ and the Alexander von Humboldt Foundation. 
is not contained in a proper complete totally geodesic submanifold of $Q_{c}^{n+p}$. We call $f$ circular if there exists a parallel orthogonal tensor field $J$ on $M$ with $J^{2}=-I$ such that the second fundamental form $A_{\xi}$ in any normal direction $\xi$ satisfies

$$
J A_{\xi}=-A_{\xi} J
$$

In this case, $M^{n}$ is a Kaehler manifold with complex structure $J$. It follows from (1.1) that $-A_{\xi}$ is similar to $A_{\xi}$, so all odd symmetric functions vanish. In particular, $f$ is minimal and thus real analytic.

The simplest examples of circular submanifolds are all orientable minimal surfaces in $Q_{c}$ and Kaehler submanifolds of $\mathbf{C}^{m}$. Other examples will be discussed later. We will also see that a Kaehler structure $J$ satisfying (1.1) is essentially unique.

It is well-known that minimal surfaces in $Q_{c}^{3}$ have isometric "associated families", cf. [14]. This concept can be generalized to circular immersions as follows: For $\theta \in S^{1}=\mathbf{R} / 2 \pi \mathbf{Z}$, consider the orthogonal parallel tensor field $J_{\theta}=e^{\theta J}=\cos \theta I+\sin \theta J$. For any normal field $\xi$, the tensor field $A_{\theta}(\xi)=$ $A_{\xi} J_{\theta}=J_{-\theta} A_{\xi}=J_{-\theta / 2} A_{\xi} J_{\theta / 2}$ is selfadjoint and $\operatorname{tr} A_{\theta}=0$, by (1.1). It is straighforward to check that $A_{\theta}$ satisfies the Gauss equations.

Lemma 1.2. With respect to the normal connection of $f$, the tensor $A_{\theta}$ satisfies the Codazzi and Ricci equations.

Proof. This follows immediately from the relations $\nabla A_{\theta}=J_{-\theta} \nabla A$ and $\left[A_{\theta}(\xi), A_{\theta}(\eta)\right]=[A(\xi), A(\eta)]$, using the Fundamental Theorem of submanifolds.

Assume $M$ is simply connected and fix the normal bundle of $f$ with its induced connection. Then by Lemma 1.2, there exists a circular immersion $f_{\theta}$ in $Q$, unique up to congruence, with second fundamental form $A_{\theta}$. We call the congruence classes of the one-parameter family $f_{\theta}$ the associated family of $f$.

In general, the associated family is nontrivial, i.e. for $\theta_{1} \not \equiv \theta_{2} \bmod \pi$, the maps $f_{\theta_{1}}$ and $f_{\theta_{2}}$ are not congruent. This is always the case for (non-totally geodesic) hypersurfaces. Note that $f_{\theta+\pi}$ and $f_{\theta}$ are congruent since $A_{\theta+\pi}=$ $-A_{\theta}$, and $-I$ is a parallel automorphism of the normal bundle. The congruence is orientation-preserving if the codimension $p$ is even. To discuss this question further we introduce the following definition: Any isometric immersion $f$ of a Kaehler manifold is called pseudoholomorphic if there exists an orthogonal tensor field $T$ on the normal bundle of $f$, parallel in the normal connection, such that

$$
A_{T \xi}=A_{\xi} J
$$

for all $\xi$. It follows that $A_{\xi} J$ must be selfadjoint, so $f$ is circular. 
Lemma 1.4. $f$ is pseudoholomorphic if and only if the complex structure of $M$ extends to a parallel complex structure $J$ of the tangent bundle of $Q$ restricted to $f$ such that

$$
A_{J \xi}=A_{\xi} J
$$

In particular, for $c=0$, pseudoholomorphic submanifolds are precisely Kaehler submanifolds of $\mathbf{R}^{n+p}=\mathbf{C}^{m}$, up to congruence.

Proof. Apply (1.3) twice to obtain $A_{T^{2} \xi}=A_{T \xi} J=-A_{\xi}=A_{-\xi}$, so $A_{\left(T^{2}+I\right) \xi}$ $=0$. The image of the parallel tensor $T^{2}+I$ is a parallel and totally geodesic subbundle of the normal bundle, which must be zero. (It is a well-known fact that otherwise $f$ is not substantial.) Therefore, $T^{2}=-I$. It follows easily with (1.5) that the complex structure thus obtained for the tangent bundle of $Q$ along $f$ is parallel.

For the second part of the lemma observe that (1.5) holds for complex submanifolds of any Kaehler manifold. Conversely, if $J$ is parallel along $f$ in $\mathbf{R}^{n+p}=\mathbf{C}^{m}$, it extends to a complex structure $J$ of $\mathbf{R}^{n+p}$, which is conjugate to the standard complex structure of $\mathbf{C}^{m}$ in the orthogonal group $O(n+p)$.

Proposition 1.6. Let $f_{\theta}$ be the associated family of the circular immersion $f$ : $M^{n} \rightarrow Q^{n+p}$. Then, $f_{\theta}$ is trivial if $f$ is pseudoholomorphic. Conversely, if $f_{\theta_{1}}$ is congruent to $f_{\theta_{2}}$ for some $\theta_{1} \not \equiv \theta_{2} \bmod \pi$, then $f$ is pseudoholomorphic.

Proof. By Lemma 1.4, we have a parallel extension $J$ of the complex structure of $M$. Define, as before, $J_{\theta}=\cos \theta I+\sin \theta J$. Now $A_{J_{\theta} \xi}=A_{\xi} J_{\theta}$, and the $f_{\theta}$ are congruent by the uniqueness part of the Fundamental Theorem of submanifolds. To prove the converse, we may assume $\theta_{1}=0<\theta_{2}=\theta<\pi$. Since $f_{\theta}$ and $f_{0}$ are congruent, there is a parallel orthogonal tensor field $S$ on the normal bundle so that $A_{S \xi}=A_{\xi} J_{\theta}$, so

$$
A_{S_{\theta} \xi}=A_{\xi} J
$$

where $\sin \theta S_{\theta}=S-\cos \theta I$. Therefore, $-A_{\xi}=A_{\xi} J^{2}=A_{S_{\theta} \xi} J=A_{S_{\theta}^{2} \xi}$, and it follows as before that $S_{\theta}^{2}=-I$. It remains to show that $S_{\theta}$ is orthogonal. The last equation is equivalent to $S^{2}-2 \cos \theta S+I=0$, and thus $S+S^{t}=$ $2 \cos \theta I$. On the other hand, $\sin ^{2} \theta S_{\theta} S_{\theta}^{t}=I-\cos \theta\left(S+S^{t}\right)+\cos ^{2} \theta I=\sin ^{2} \theta I$. Now, $J=S_{\theta}$ is a parallel complex structure on the normal bundle, and $f$ is pseudoholomorphic by (1.7).

The last proposition says that the congruence classes of associated immersions $f_{\theta}$ are parametrized precisely by the circle $\mathbf{R} / \pi \mathbf{Z}$, unless $f$ is pseudoholomorphic when they all coincide, which is impossible for odd codimension $p$.

In spaces of nonzero constant curvature, minimal surfaces are actually the only circular submanifolds. 
Proposition 1.8. Let $f: M^{n} \rightarrow Q_{c}^{n+p}$ be circular and $c \neq 0$. Then $n=2$.

Proof. For any $u, v, w, z$ we have $\langle R(u, v) w, z\rangle=\langle R(u, v) J w, J z\rangle$. It follows from the Gauss equations and (1.1) that for an orthonormal frame $e_{1}, \cdots, e_{2 m}$ with $J e_{2 k-1}=e_{2 k}$, the Ricci curvature satisfies

$$
(2 m-1) c-\sum_{j=1}^{2 m}\left|\alpha\left(e_{1}, e_{j}\right)\right|^{2}=\operatorname{Ric}\left(e_{1}, e_{1}\right)=c-\sum_{j=1}^{2 m}\left|\alpha\left(e_{1}, e_{j}\right)\right|^{2}
$$

Here $\alpha$ denotes the normal valued second fundamental form. The first equality in (1.9) holds already whenever $f$ is minimal. We conclude $2 m=n=2$.

The situation is quite different in euclidean spaces. In the next section we will classify circular hypersurfaces. The following result shows that these examples give rise to an abundance of circular submanifolds with higher codimension.

Proposition 1.10. Let $M$ be circular in $\mathbf{R}^{m}$, and $N$ a complex submanifold of $M$. Then $N$ is circular in $\mathbf{R}^{m}$.

Proof. This follows from (1.1) and the fact that $N$ is a Kaehler submanifold of $M$.

Another important observation is this: Let $f_{i}: M^{n} \rightarrow \mathbf{R}^{m_{i}}\left(M^{2} \rightarrow S^{m_{i}}\right)$ be circular, $i=1,2$. Then $\alpha_{1} f_{1} \oplus \alpha_{2} f_{2}: M \rightarrow \mathbf{R}^{m_{1}+m_{2}}\left(S^{m_{1}+m_{2}+1}\right)$ is circular, where $\alpha_{1}^{2}+\alpha_{2}^{2}=1$. In particular, we have the 2-parameter family $f(\varphi, \theta)=$ $\cos \varphi f_{\theta} \oplus \sin \varphi f_{\theta+\pi / 2}$ of circular immersions in $\mathbf{R}^{2 m}$, where $f_{\theta}$ is the associated family of a circular immersion $f: M \rightarrow \mathbf{R}^{m}$, with $M$ simply connected. The above family was first considered in [15] for minimal $M^{2}$ in $\mathbf{R}^{3}$.

We will now generalize a basic result of Calabi; cf. [17, Theorem 4, p. 148].

Theorem 1.11. Let $M^{n}$ be a simply connected Kaehler manifold, and suppose there exists a circular immersion $f: M^{n} \rightarrow \mathbf{R}^{n+p}$. Then the set of all noncongruent circular immersions of $M$ into euclidean spaces contains a unique ( pseudo)holomorphic representative.

Let us first give an explicit description of associated families for circular immersions in euclidean spaces. In a slightly more general form, this will also be important later.

Lemma 1.12. Let $f: M^{n} \rightarrow \mathbf{R}^{n+p}$ be an isometric immersion of a riemannian manifold, and let $T$ be an orthogonal parallel tensor field on $M$. Then the 1-form $d f \circ T=f_{*} \circ T$ on $M$ with values in $\mathbf{R}^{n+p}$ is closed if and only if $T$ satisfies

$$
A_{\xi} T=T^{t} A_{\xi}
$$

for all normal vectors $\xi$. 
Proof. We have $d\left(f_{*} \circ T\right)(X, Y)=\tilde{\nabla}_{X} f_{*} T Y-\tilde{\nabla}_{Y} f_{*} T X-f_{*} T[X, Y]$ along $f$ in $\mathbf{R}^{n+p}$. Taking tangent and normal components, we obtain $\tilde{\nabla}_{X} f_{*} T Y=$ $f_{*} \nabla_{X} T Y+\alpha(X, T Y)=f_{*} T \nabla_{X} Y+\alpha(X, T Y)$, where $\alpha$ is the second fundamental form with values in the normal bundle. Thus, $d\left(f_{*} \circ T\right)(X, Y)=$ $\alpha(X, T Y)-\alpha(Y, T X)$ vanishes precisely when (1.13) holds.

Proposition 1.14. Let $f: M^{n} \rightarrow \mathbf{R}^{n+p}$ be circular, $M$ simply connected. Then the associated family $f_{\theta}$ is explicitly given by the line integral

$$
f_{\theta}(x)=\int_{x_{0}}^{x} f_{*} \circ J_{\theta},
$$

where $x_{0}$ is any fixed point in $M$.

Proof. By Lemma 1.12, $f_{\theta}$ is well defined. Clearly,

$$
f_{\theta *}=f_{*} \circ J_{\theta},
$$

which shows that $f_{\theta}$ is isometric, and the tangent (normal) spaces of $f$ and $f_{\theta}$ at $x$ are parallel in $\mathbf{R}^{n+p}$ for all $x \in M$, i.e. all $f_{\theta}$ have the same Gauss map; cf. §3. In particular, $f_{\theta}$ and $f$ have the same normal connection. Using (1.16), the second fundamental form of $f_{\theta}$ is given by

$$
\begin{aligned}
\alpha_{\theta}(X, Y) & =\tilde{\nabla}_{X} f_{\theta *} Y-f_{\theta *} \nabla_{X} Y=\tilde{\nabla}_{X} f_{*} J_{\theta} Y-f_{*} J_{\theta} \nabla_{X} Y \\
& =\tilde{\nabla}_{X} f_{*} J_{\theta} Y-f_{*} \nabla_{X} J_{\theta} Y=\alpha\left(X, J_{\theta} Y\right),
\end{aligned}
$$

so $A_{\theta}=A J_{\theta}$. This completes the argument.

Proof of Theorem 1.11. Uniqueness follows from Calabi's rigidity theorem for Kaehler submanifolds of $\mathbf{C}^{k}$; see [5]. For existence, consider $\mathbf{C}^{n+p}=$ $\mathbf{R}^{n+p} \oplus \mathbf{R}^{n+p}$ with the usual complex structure $J(u, v)=(-v, u)$. Now $g$ : $M \rightarrow \mathbf{C}^{n+p}$,

$$
g=\frac{1}{\sqrt{2}} f \oplus \frac{1}{\sqrt{2}} f_{\pi / 2},
$$

will be holomorphic, since the Jacobian $g_{*}$ is complex linear by (1.16).

Observe that $g=f(\pi / 4,0)$ belongs to the 2-parameter family of $f$ discussed before. By uniqueness, it follows that all $f(\pi / 4, \theta)$ are congruent, which can also be seen directly by use of Proposition 1.14.

We conclude this section with some remarks. In [8], we had defined associated families for certain minimal hypersurfaces. Circular hypersurfaces will turn out to be a special case; see $\S 2$.

Pseudoholomorphic surfaces $f: M^{2} \rightarrow S^{2 k}$ were first studied by Calabi [6]. By definition, they induce a map into the hermitian symmetric space $\mathfrak{S}_{k}=$ $S O(2 k+1) / U(k)$ of all oriented hyperplanes in $\mathbf{R}^{2 k+1}$ with complex structure, which is holomorphic. Conversely any holomorphic curve in $\mathfrak{S}_{k}$ projects to a pseudoholomorphic surface in $S^{2 k}$; cf. [3], [7], [10], [16]. A main global 
result is that any minimal immersion of the sphere $S^{2}$ (not necessarily with constant curvature) in $S^{2 k}$ is pseudoholomorphic, and actually rigid. Superminimal surfaces in spheres, in the sense of [4], are pseudoholomorphic in general only for codimension 2 .

Real Kaehler submanifolds of higher dimension do not seem to have received very much attention in the literature. For some significant contributions, we refer to [19], [1], and [11].

We finally mention another interesting fact that was obtained after completion of this paper, due to the first author and L. Rodriguez: Any minimal immersion of a Kaehler manifold in euclidean space is circular, so "circular" and "minimal real Kaehler" mean the same in euclidean spaces, although this is not obvious; cf. [9].

\section{Real Kaehler hypersurfaces}

Let $f: M^{2 m} \rightarrow \mathbf{R}^{2 m+1}$ be an isometric immersion of a Kaehler manifold with real codimension 1 . We will essentially give a local classification of such hypersurfaces, a question which was raised in [19], and answered if the ambient space has nonzero constant curvature. It is a simple fact due to Takahashi [20] (see also [1]) that the Gauss map $\varphi: M \rightarrow S^{2 m}$ has rank $\leqslant 2$ everywhere. Since the flat case is not interesting, let us assume $\operatorname{rank} \varphi=2$ is constant. The relative nullity bundle $\Delta=\operatorname{ker} \varphi_{*}$ and its orthogonal complement $\Delta^{\perp}$ are invariant under the complex structure $J$ of $M$.

Proposition 2.1. Let $f: M^{2 m} \rightarrow \mathbf{R}^{2 m+1}$ be an isometric immersion without flat points. Then, at least locally, $M$ is Kaehler if and only if the Gauss map $\varphi$ has constant rank 2 and its spherical image is a pseudoholomorphic surface (not necessarily substantial).

Proof. Since $M$ has no flat points, $\operatorname{dim} \Delta^{\perp}=2$ everywhere. Notice first that $\Delta_{x}\left(\Delta_{x}^{\perp}\right)$ are parallel in $\mathbf{R}^{2 m+1}$ to the normal (tangent) space of the Gauss image $V^{2}=\varphi(M)$ in $S^{2 m}$ at $y=\varphi(x)$. Let $Y, Z$ be tangent fields, and $\xi$ a normal field of $V^{2}$. Consider $\tilde{Y}, \bar{Z} \in \Delta^{\perp}, \bar{\xi} \in \Delta$ such that $\tilde{Y}$ is $\varphi$-related to $Y$, and $\bar{Z}=Z \circ \varphi, \bar{\xi}=\xi \circ \varphi$. Now in $\mathbf{R}^{2 m+1}$,

$$
\tilde{\nabla}_{\tilde{Y}} \bar{\xi}=\left(\tilde{\nabla}_{Y} \xi\right) \circ \varphi
$$

Thus

$$
\left\langle\nabla_{\tilde{Y}} \bar{\xi}, \bar{Z}\right\rangle=\left\langle\tilde{\nabla}_{\tilde{Y}} \bar{\xi}, \bar{Z}\right\rangle=\left\langle\tilde{\nabla}_{Y} \xi, Z\right\rangle=-\left\langle A_{\xi} X, Z\right\rangle,
$$

where $\nabla$ is the connection in $M$, and $A_{\xi}$ is the second fundamental form of $V^{2}$ in direction $\xi$. If $M$ is Kaehler with complex structure $J$, define complex structures $J$ on $V^{2}$ and its normal bundle in $S^{2 m}$ by using the above 
identification with $\Delta^{\perp}$ and $\Delta$, which are invariant under $J$. It follows immediately from (2.2) that $J$ is parallel in the normal connection of $V^{2}$. By (2.3),

$$
-\left\langle A_{\xi} Y, Z\right\rangle=\left\langle\nabla_{\tilde{Y}} \bar{\xi}, \bar{Z}\right\rangle=\left\langle\nabla_{\tilde{Y}} J \bar{\xi}, J \bar{Z}\right\rangle=-\left\langle A_{J \xi} Y, J Z\right\rangle,
$$

therefore $J A_{J \xi}=-A_{\xi}$, so $A_{J \xi}=J A_{\xi}$, and $V^{2}$ is pseudoholomorphic. Conversely, if $V^{2}$ is pseudoholomorphic, reverse the argument to obtain an almost complex structure on $M$ which is parallel.

For the following result, we recall from [8] the Gauss representation of hypersurfaces with constant relative nullity $n-2$. Let $g: V^{2} \rightarrow S^{2 m}$ be any isometric immersion with normal bundle $\Lambda$ along $g, \gamma$ an arbitrary "support" function on $V^{2}$. Then, the map $\psi: \Lambda \rightarrow \mathbf{R}^{2 m+1}$,

$$
\psi(y, w)=\gamma g(y)+\nabla \gamma+w,
$$

parametrizes a hypersurface with constant relative nullity $n-2$, wherever it is regular. Here the sums are taken in $\mathbf{R}^{2 m+1}$, and $\nabla \gamma$ is the gradient of $\gamma$ in $V$. Conversely, all such hypersurfaces can be obtained this way locally. By combining this with Proposition 2.1, we now have the main result of this section.

Theorem 2.5. The real Kaehler hypersurfaces in $\mathbf{R}^{2 m+1}$, without flat points, are precisely given by the Gauss representation (2.4), in terms of any spherical pseudoholomorphic surface and an arbitrary support function.

Here again we do not require the pseudoholomorphic surfaces to be substantial. If the Gauss image is substantial in $S^{2 k} \subset S^{2 m}$, then locally, the Kaehler hypersurface has totally geodesic flat factors of dimension $2(m-k)$, i.e., it is reducible.

Corollary 2.6. A real Kaehler hypersurface in $\mathbf{R}^{2 m+1}$ is circular if and only if its support function $\gamma$ in the representation (2.4) satisfies $\Delta \gamma+2 \gamma=0$, on the Gauss image.

Proof. The immersion is circular iff it is minimal, and the claim follows from [8].

Notice also that the Gauss map of a real Kaehler hypersurface is antiholomorphic precisely in the circular case.

As a global application, we give a short proof of an interesting result in [1].

Corollary 2.7 (K. Abe). Any complete real Kaehler hypersurface $M^{2 m}$ in $\mathbf{R}^{2 m+1}$ without flat points is a cylinder $V^{2} \times \mathbf{R}^{2 m-2}$.

Proof. The Gauss representation (2.4) can be taken globally, since $M$ is complete. It suffices to show that $V^{2}$ is totally geodesic. If not, then the first normal space has dimension 2 almost everywhere, since $V^{2}$ is pseudoholomorphic. This leads to a contradiction as in the proof of Theorem 2.5 in [8]. 
The conclusion in Abe's theorem remains true also if $M$ has nonnegative scalar curvature by Hartman's Theorem [12], or if the immersion of $M$ is real analytic. To prove the latter statement, observe that the above argument actually applies to the open subset of nonflat points in the complete manifold $M$.

\section{Submanifolds with the same Gauss map}

The Gauss map of an isometric immersion $f: M^{n} \rightarrow \mathbf{R}^{n+p}$ is the induced map $\varphi: M^{n} \rightarrow G_{n p}$, the Grassmannian of all nonoriented $n$-planes through the origin in $\mathbf{R}^{n+p}$, by parallel translating the tangent space $f_{*} T_{x} M$ to the origin. Let $\tilde{f}: M^{n} \rightarrow \mathbf{R}^{n+p}$ be another isometric immersion. Then $f$ and $\tilde{f}$ are said to have congruent Gauss maps if there exists an orthogonal transformation $\tau \in O(n+p)$ such that $\tilde{\varphi}=\tau \circ \varphi$, where $\tau$ acts naturally on $G_{n p}$. We will now start to discuss the problem when $f$ and $\tilde{f}$ are congruent if their Gauss maps are congruent. It is clearly sufficient to assume that $f$ and $\tilde{f}$ have the same - Gauss map $\varphi=\tilde{\varphi}$, i.e. the tangent (normal) spaces of $f$ and $\tilde{f}$ at $x$ are parallel in $\mathbf{R}^{n+p}$ for all $x \in M$. Examples for this situation are the associated families of circular immersions; cf. Proposition 1.14.

Lemma 3.1. Suppose isometric immersions $f, \tilde{f}: M^{n} \rightarrow \mathbf{R}^{n+p}$ have the same Gauss map. Then the orthogonal tensor field $T=f_{*}^{-1} \circ \tilde{f}_{*}$ is parallel on $M$, and the second fundamental forms along $f$ and $\tilde{f}$ satisfy

$$
\tilde{A}=A \circ T=T^{-1} \circ A,
$$

where tangent and normal spaces along $f$ and $\tilde{f}$ are identified by parallel transport in $\mathbf{R}^{n+p}$.

Proof. Let $P_{x}: T_{\tilde{f}(x)} \mathbf{R}^{n+p} \rightarrow T_{f(x)} \mathbf{R}^{n+p}$ denote parallel transport. For any vector field $U$ along $\tilde{f}$ we have the vector field $P U$ along $f$ given by $(P U)_{x}=P_{x} U_{x}$, and clearly $\tilde{\nabla}_{X} P U=P \tilde{\nabla}_{X} U$ for tangent fields $X$ on $M$, where $\tilde{\nabla}$ is the connection of $\mathbf{R}^{n+p}$. Now

$$
\begin{aligned}
\left\langle\nabla_{X} T Y, Z\right\rangle & =\left\langle\nabla_{X} f_{*}^{-1} P \tilde{f}_{*} Y, Z\right\rangle=\left\langle f_{*} \nabla_{X} f_{*}^{-1} P \tilde{f}_{*} Y, f_{*} Z\right\rangle \\
& =\left\langle\tilde{\nabla}_{X} P \tilde{f}_{*} Y, f_{*} Z\right\rangle=\left\langle P \tilde{\nabla}_{X} \tilde{f}_{*} Y, f_{*} Z\right\rangle \\
& =\left\langle P \tilde{f}_{*} \nabla_{X} Y, f_{*} Z\right\rangle=\left\langle T \nabla_{X} Y, Z\right\rangle,
\end{aligned}
$$

and $T$ is parallel. By taking normal components, we obtain for a normal field $\xi$ along $f$,

$$
\begin{aligned}
\left\langle\tilde{A}_{P^{-1} \xi} X, Y\right\rangle & =\left\langle\tilde{f}_{*} \tilde{A}_{P^{-1} \xi} X, Y\right\rangle=-\left\langle\tilde{\nabla}_{X} P^{-1} \xi, \tilde{f}_{*} Y\right\rangle=\left\langle P^{-1} \xi, \tilde{\nabla}_{X} \tilde{f}_{*} Y\right\rangle \\
& =\left\langle\xi, P \tilde{\nabla}_{X} \tilde{f}_{*} Y\right\rangle=\left\langle\xi, \tilde{\nabla}_{X} P \tilde{f}_{*} Y\right\rangle=-\left\langle\tilde{\nabla}_{X} \xi, P \tilde{f}_{*} Y\right\rangle \\
& =\left\langle f_{*} A_{\xi} X, P \tilde{f}_{*} Y\right\rangle=\left\langle A_{\xi} X, T Y\right\rangle,
\end{aligned}
$$

which completes the argument, since $A$ and $\tilde{A}$ are selfadjoint. 
The last lemma motivates a generalization of the concept of associated families. Let $f: M^{n} \rightarrow \mathbf{R}^{n+p}$ be any isometric immersion, $M^{n}$ simply connected. Consider the set $K$ of orthogonal parallel tensor fields $T$ on $M$ that satisfy

$$
A T=T^{-1} A
$$

for any normal direction. Given $T \in K$, we can define the associated immersion $f_{T}: M^{n} \rightarrow \mathbf{R}^{n+p}$ completely analogously as in $\S 1$. Therefore, $f_{T}$ is isometric, with second fundamental form (3.3), it has the same Gauss map as $f$, and it is explicitly given by

$$
f_{T}(x)=\int_{x_{0}}^{x} f_{*} \circ T
$$

We call the set $f_{\Re}$ of all congruence classes of immersions associated with $f$ the associated family of $f$. We conclude from Lemma 3.1 that equivalently, $f_{\Re}$ is precisely the set of all isometric immersions with same Gauss map as $f$, up to congruences.

Our main goal is to determine the associated family $f_{\Re}$. This will be done in various steps. In this section, we show that $K$ is a group and compute its structure.

Lemma 3.5. The tangent bundle of $M$ splits uniquely as

$$
T M=\Gamma_{0} \oplus \Gamma_{1} \oplus \cdots \oplus \Gamma_{r},
$$

where $\Gamma_{i}$ are mutually orthogonal A-invariant parallel subbundles, $\Gamma_{0}$ is the maximal totally geodesic subbundle, and $\Gamma_{i}$ is irreducible as an A-invariant parallel bundle for $1 \leqslant i \leqslant r$. Any parallel A-invariant subbundle $\Gamma$ is the sum of $\Gamma \cap \Gamma_{0}$ and some of the factors $\Gamma_{1}, \cdots, \Gamma_{r}$.

We will also refer to (3.6) as the A-decomposition for $f$.

Proof. Clearly, $\Gamma_{0}$ is unique. It remains to show that any $A$-irreducible parallel subbundle $\Gamma \subset \Gamma_{1} \oplus \cdots \oplus \Gamma_{r}$ must be a summand. The bundle projections $\pi_{i}: \Gamma_{1} \oplus \cdots \oplus \Gamma_{r} \rightarrow \Gamma_{i}$ are parallel and commute with $A$. Since $\Gamma$ and $\Gamma_{i}$ are $A$-irreducible, $\pi_{i} \mid \Gamma$ is an isomorphism or 0 . If $\pi_{i} \mid \Gamma$ and $\pi_{j} \mid \Gamma$ are isomorphisms for $i \neq j$, then $\chi=\pi_{j} \circ\left(\pi_{i} \mid \Gamma\right)^{-1}$ is a bundle isomorphism $\Gamma_{i} \rightarrow \Gamma_{j}$ commuting with $A$. Take a point $x \in M$ such that $A u \neq 0$ for $u \in \Gamma_{i \mid x}$. Now near $x$, we have a splitting $U=U^{\prime} \times U_{i} \times U_{j}$ in $M$, where $U_{i}, U_{j}$ are local integral manifolds of $\Gamma_{i}, \Gamma_{j}$. It follows that on $U$, the immersion $f$ splits as $f=f^{\prime} \times f_{i} \times f_{j}$; cf. [18, Lemma, p. 163]. Thus we find a normal vector $\xi$ at $x$ with $A_{\xi} u \neq 0$, but $A_{\xi} \mid \Gamma_{j}=0$. This is a contradiction since $0=A_{\xi} \chi u=\chi A_{\xi} u$ $\neq 0$.

Let us call a parallel $A$-invariant subbundle $\Gamma$ of $T M$ circular if there exists a parallel complex structure $J$ for $\Gamma$ so that $A J=-J A$ on $\Gamma$. This implies that any leaf of $\Gamma$ is a Kaehler manifold restricted to which $f$ is circular. 
Lemma 3.7. Suppose $\Gamma_{\mu_{1}} \oplus \cdots \oplus \Gamma_{\mu_{s}} \subset T M, 1 \leqslant \mu_{k} \leqslant r$, is circular. Then each $\Gamma_{\mu_{k}}$ is invariant under $J$ (and circular).

Proof. Let $k \neq l$ and $u \in \Gamma_{\mu_{k}}, v \in \Gamma_{\mu_{i}}$. Then $R(u, J v) J v=J R(u, J v) v$, so $\langle R(u, J v) J v, u\rangle=-\langle R(u, J v) v, J u\rangle^{\prime}=0$. Therefore, by the Gauss equations,

$$
|\alpha(u, J v)|^{2}=\langle\alpha(u, u), \alpha(J v, J v)\rangle=-\langle\alpha(u, u), \alpha(v, v)\rangle=0,
$$

since $A$ and $J$ anticommute. Thus, $\alpha(u, J v)=\alpha(J u, v)=0$. Observe that $J \Gamma_{\mu_{k}}$ is parallel and $A$-invariant. Moreover, $J \Gamma_{\mu_{k}}$ is $A$-irreducible, since $\Gamma_{\mu_{k}}=$ $J\left(J \Gamma_{\mu_{k}}\right)$ is $A$-irreducible. We conclude from Lemma 3.5 that $J \Gamma_{\mu_{k}}=\Gamma_{\mu_{l}}$ for some $l$. If $l \neq k, \alpha(J u, v)=0$ would imply $\Gamma_{\mu} \subset \Gamma_{0}$, so $l=k$.

Before we proceed, let us discuss a few simple facts about parallel orthogonal tensor fields on a simply connected riemannian manifold $N$. The group of such fields $T$ is canonically isomorphic to the centralizer of the holonomy group $\Phi$ in the orthogonal group of the tangent space of $N$ at a given point $p$. Any linear subspace invariant under $\Phi$ extends to a parallel subbundle of $T N$. Now $T$ gives rise to a parallel splitting $T N=\Delta_{+} \oplus \Delta_{-} \oplus \Delta_{1} \oplus \cdots \oplus \Delta_{m}$, where $\Delta_{ \pm}$are the \pm 1 eigenspaces, and $\Delta_{1}, \cdots, \Delta_{m}$ are the eigenspaces for the distinct nonconjugate complex eigenvalues $e^{i \theta_{\mu}}$ of $T$, where $0<\theta_{\mu}<\pi$ are distinct. The $\Delta_{\mu}$ have a parallel complex structure

$$
J_{\mu}=\frac{1}{2 \sin \theta_{\mu}}\left(T-T^{-1}\right)
$$

that commutes with $T$. Thus $J=J_{1} \oplus \cdots \oplus J_{m}$ is a parallel complex structure for $\Delta=\Delta_{1} \oplus \cdots \oplus \Delta_{m}$ commuting with $T$, and $T=e^{\theta_{1} J_{1}} \oplus \cdots \oplus e^{\theta_{m} J_{m}}$.

Lemma 3.8. Any $T \in K$ leaves all $\Gamma_{i}$ in the A-decomposition invariant.

Proof. We need to show that any $T \in K$ leaves the splitting (3.6) invariant. Clearly $T \Gamma_{0}$ is parallel and $A T \Gamma_{0}=T^{-1} A \Gamma_{0}=0$, so $T \Gamma_{0} \subset \Gamma_{0}$ by definition. Now, we can assume $T \mid \Gamma_{0}=$ id. Consider the parallel orthogonal splitting $T M=\Delta_{+} \oplus \Delta_{-} \oplus \Delta$. We have $\Gamma_{0} \subset \Delta_{+}$. This splitting is invariant under $A$. Simply observe that for $u \in \Delta_{+}\left(\Delta_{-}\right), T A u=A T^{-1} u=(-) A u$. Now by uniqueness in Lemma 3.5, both $\Delta_{+}$and $\Delta_{-}$are direct sums of factors in (3.6), which are therefore trivially left invariant under $T$. Consider $\Delta$ which is the sum of the remaining $\Gamma_{j}$. Now $S=\frac{1}{2}\left(T+T^{-1}\right)$ commutes with $A$, since $T^{-1} \in K$. We have $S=\cos \theta_{1} I_{1} \oplus \cdots \oplus \cos \theta_{m} I_{m}$, where $I_{\mu}$ is the identity on $\Delta_{\mu}$, so $A$ leaves $\Delta_{\mu}$ invariant, since the eigenvalues $\cos \theta_{\mu}$ are mutually distinct. On the other hand, $T-T^{-1}$ anticommutes with $A$, and the restriction $J_{\mu}$ of $\left(T-T^{-1}\right) / 2 \sin \theta_{\mu}$ is a complex structure for $\Delta_{\mu}$. By Lemma $3.5, \Delta_{\mu}=\Gamma_{\mu_{1}}$ $\oplus \cdots \oplus \Gamma_{\mu_{s}}$, and by Lemma 3.7, all $\Gamma_{\mu_{k}}$ are invariant under $J_{\mu}$. But $T \mid \Delta_{\mu}=$ $\cos \theta_{\mu} I_{\mu}+\sin \theta_{\mu} J_{\mu}$, so $T$ leaves all $\Gamma_{\mu_{k}}$ invariant. 
Going back to the $A$-decomposition for $f$, we now consider the subsets $K_{i} \subset K$ consisting of all $T \in K$ leaving $\Gamma_{j}$ fixed for all $j \neq i$. It is clear that each $K_{i}$ contains at least two elements which are \pm id on $\Gamma_{i}$. Furthermore, $K_{0}$ is the group $O\left(m_{0}\right)=O\left(\Gamma_{0}\right)$. We have a canonical injective map

$$
\gamma: K_{0} \times K_{1} \times \cdots \times K_{r} \rightarrow K
$$

given by $\gamma\left(T_{0}, T_{1}, \cdots, T_{r}\right)-T_{0} T_{1} \cdots T_{r}$. We conclude from Lemma 3.8 that $\gamma$ is onto and thus a one-to-one correspondence.

Lemma 3.10. If $\Gamma_{i}$ is circular, $1 \leqslant i \leqslant r$, with complex structure $J$, then $K_{i}$ is the circle $e^{\theta J}, \theta \in \mathbf{R} / 2 \pi \mathbf{Z}$.

Proof. Clearly, $e^{\theta J} \in K_{i}$. Conversely, let $T \in K_{i}$. As we have seen, $T$ can only have one pair of conjugate eigenvalues which we may assume to be not real. Then $T=e^{\varphi J^{\prime}}$ for some parallel complex structure $J^{\prime}$ on $\Gamma_{i}$ which also anticommutes with $A$. It remains to show that $J^{\prime}= \pm J$. The orthogonal parallel tensor field $L=J J^{\prime}$ on $\Gamma_{i}$ commutes with $A$. Since $\Gamma_{i}$ is $A$-irreducible, it follows as before that $L$ has only one pair of conjugate eigenvalues $e^{i \theta}$, $0 \leqslant \theta \leqslant \pi$. If $\theta=0$ or $\theta=\pi$, then $L= \pm I$, and we are done. So suppose $0<\theta<\pi$. Then $J^{*}=\left(L-L^{-1}\right) / 2 \sin \theta$ is a parallel complex structure for $\Gamma_{i}$, which commutes with $A$, i.e. $\alpha\left(J^{*} u, v\right)=-\alpha\left(u, J^{*} v\right)$. But now we obtain for the sectional curvatures,

$$
\begin{aligned}
\langle R(u, v) v, u\rangle & =\left\langle R\left(J^{*} u, J^{*} v\right), v, u\right\rangle \\
& =\left\langle\alpha\left(J^{*} u, u\right), \alpha\left(J^{*} v, v\right)\right\rangle-\left\langle\alpha\left(J^{*} u, v\right), \alpha\left(J^{*} v, u\right)\right\rangle \\
& =\left|\alpha\left(J^{*} u, v\right)\right|^{2} \geqslant 0
\end{aligned}
$$

for all $u, v \in \Gamma_{i}$. On the other hand, the trace of $\alpha$ vanishes on $\Gamma_{i}$. It is well-known that this implies the Ricci tensor is $\leqslant 0$ on $\Gamma_{i}$, with equality holding iff $\alpha=0$. This is not possible, since $i \geqslant 1$.

We summarize our results so far as follows:

Proposition 3.11. The set $K$ is a compact subgroup of the orthogonal group, and

$$
K=O(m) \times S^{1} \times \cdots \times S^{1} \times \mathbf{Z}_{2} \times \cdots \times \mathbf{Z}_{2},
$$

where $O(m)=O\left(\Gamma_{0}\right)$, each $S^{1}$-factor corresponds to a circular factor $\Gamma_{i}$, and the $\mathbf{Z}_{2}=\{I,-I\}$ correspond to the remaining factors in the A-decomposition.

\section{The congruence problem}

We now turn to a discussion of the question when the associated immersions corresponding to different elements in the group $K$ are congruent in euclidean space. 
With the notations of $\S 3$, it is clear that $f_{T}$ is congruent to $f$ (we write $f_{T} \sim f$ for short) if $T \in K_{0}$. It remains to analyze $\tilde{K}=K / K_{0}=K_{1} \times \cdots \times K_{r}$. Let $T_{1}, T_{2} \in \tilde{K}$. By (3.4), $f_{T_{1}} \sim f_{T_{2}}$ iff $A_{\xi} T_{1}=A_{S \xi} T_{2}$ for some parallel orthogonal field $S$ on $\Lambda$ along $f$. Thus $f_{T_{1}} \sim f_{T_{2}}$ iff $f_{T_{1} T_{2}^{-1}} \sim f$, and it suffices to consider $T_{1}=T$ and $T_{2}=$ id. Now all $T \in \tilde{K}$ with $f_{T} \sim f$ form a subgroup $H \subset K$, and $T \rightarrow f_{T}$ is a one-to-one correspondence between the group $\Re=\tilde{K} / H$ and the associated family $f_{\Re}$ of all congruence classes of immersions associated with $f$. We will now determine the structure of the group $\Omega$.

We may always assume that $f$ is substantial. Recall the $A$-splitting $T M=\Gamma_{0}$ $\oplus \Gamma_{1} \oplus \cdots \oplus \Gamma_{r}$. Each $\Gamma_{i}$ gives rise to a subset $N_{i}$ of the normal bundle $\Lambda$ along $f$, where at a point $x \in M, N_{i}(x)=\operatorname{span}\left\{\alpha(u, v) \mid u, v \in \Gamma_{i}(x)\right\}$, i.e. $N_{i}$ is the "first normal space of $\Gamma_{i}$ ". Clearly $N_{0}=0$, and for all $1 \leqslant i \leqslant r, N_{i} \neq 0$ somewhere. Let us consider an orthogonal splitting

$$
\Lambda=\Lambda_{1} \oplus \cdots \oplus \Lambda_{s}
$$

such that:

(1) the $\Lambda_{j}$ are parallel subbundles,

(2) if $\pi_{j}$ denotes the projection on $\Lambda_{j}$, then $N_{i} \subset \Lambda_{j}$ everywhere whenever $\pi_{j} N_{i}(x) \neq 0$ for some $x \in M$,

(3) the splitting has a maximal number of factors.

Lemma 4.2. Each $\Lambda_{j}$ in (4.1) contains at least one $N_{i}$, and this splitting is unique.

Proof. The first part is clear, since $f$ is substantial. To prove uniqueness, suppose $\Lambda=\Lambda_{1}^{\prime} \oplus \cdots \oplus \Lambda_{s}^{\prime}$. It is sufficient to show that if $N_{i} \subset \Lambda_{j} \cap \Lambda_{k}^{\prime}$, then $\Lambda_{j}=\Lambda_{k}^{\prime}$. Otherwise, say $\Lambda_{j}=\left(\Lambda_{j} \cap \Lambda_{k}^{\prime}\right) \oplus \Omega$ splits orthogonally with $\Omega \neq 0$ parallel, and this would provide a finer splitting of $\Lambda$ satisfying conditions (1) and (2), in contradiction to (3).

We call (4.1) the $\alpha$-splitting of $\Lambda$ along $f$. Note that each $\Lambda_{k}$ is precisely the parallel hull of all $N_{i} \subset \Lambda_{k}$, i.e. the smallest parallel subbundle containing them. This follows with the fact that $f$ is substantial. We say $\Gamma_{i}$ is simple if $N_{i} \subset \Lambda_{k}$ implies $N_{j} \subset \Lambda_{k}^{\perp}$ for $j \neq i$. At the end of this section, we will give examples where $\Gamma_{i}$ is not simple.

Lemma 4.3. Any circular $\Gamma_{i}$ is simple.

Proof. The argument depends very much on the fact that all leaves of $\Gamma_{i}$ are minimal and thus real analytic. In fact, if an analytic submanifold $W \subset \mathbf{R}^{q}$ is substantial, then the normal subspace spanned by all derivatives of the image of the second fundamental form is the whole normal space on an open dense subset. To prove this, observe, for example, that height functions on $\mathbf{R}^{q}$ restricted to $W$ are analytic. Therefore, the parallel hull of the first normal space of any open subset $V \subset W$ is the restriction of the parallel hull of the 
first normal space of $W$. Now, as the leaves $L_{i}$ of $\Gamma_{i}$ near any point $x \in M$ are factors of a local isometric splitting of the immersion $f$, the bundle spanned by $\Gamma_{i}$ and the parallel closure of $N_{i}$ is locally, and then also globally, parallel along $f$ in $\mathbf{R}^{n+p}$. Therefore, we have an orthogonal splitting $\mathbf{R}^{n+p}=\mathbf{R}^{m_{i}} \oplus \mathbf{R}^{n+p-m_{i}}$ so that each $L_{i}$ is contained in an affine subspace $E^{m_{i}}$ parallel to $\mathbf{R}^{m_{i}}$, and any leaf $L_{j}$ of $\Gamma_{j}, j \neq i$, is contained in an affine subspace orthogonal to $\mathbf{R}^{m_{i}}$. In particular, $\Gamma_{i}$ is simple.

Consider now the group $L$ of all orthogonal parallel tensor fields $S$ on $\Lambda$ such that $A_{S \xi}=A_{\xi} T$ for some $T \in \tilde{K}$. It is straightforward to check that $L$ is canonically isomorphic to $H$.

Lemma 4.4. The group L leaves the $\alpha$-splitting invariant.

Proof. Let $S \in L$. We claim the parallel bundles $\pi_{j} S \Lambda_{i}$ are everywhere orthogonal to all $N_{k}$ if $i \neq j$. This is only nontrivial for $N_{k} \subset \Lambda_{j}$. But whenever $\xi \in \Lambda_{i}$ and $u \in \Gamma_{k}$, we have $A_{\pi_{j} S \xi} u=A_{\xi} T u+A_{\pi_{j} S_{\xi}-S_{\xi}} u=0$. Thus $\pi_{j} S \Lambda_{i}=0$, since $f$ is substantial.

For any $\Lambda_{k}$ in the $\alpha$-splitting, consider the direct sum $B_{k}$ of all $\Gamma_{i}$ such that $N_{i} \subset \Lambda_{k}$.

Lemma 4.5. If $T \in H$ and $B_{k}$ is not circular, then $T \mid B_{k}= \pm I$.

Proof. Let $B_{k}^{ \pm}$be the sum of all $\Gamma_{i}$ with $T \mid \Gamma_{i}= \pm I$, so $B_{k}=B_{k}^{+} \oplus B_{k}^{-}$. There exists $S \in L$ such that $A_{S \xi}=A_{\xi} T$ for all $\xi$, and $A_{S \xi}= \pm A_{\xi}$ whenever $\xi \in N_{i}$ and $\Gamma_{i} \subset B_{k}^{ \pm}$. We claim this implies $S= \pm I$ on $N_{i}$. Otherwise, say when $\Gamma_{i} \subset B_{k}^{-}$, we find $\xi_{0} \in N_{i}$ such that the projection of $S \xi_{0}+\xi_{0}$ on $N_{i}$ is nonzero, since $S$ is orthogonal, and hence $A_{S \xi_{0}+\xi_{0}} \not \equiv 0$, which is a contradiction. Now $\Lambda_{k}=\Lambda_{k}^{+} \oplus \Lambda_{k}^{-}$is an orthogonal parallel splitting, where $\Lambda_{k}^{+}$are the \pm 1 eigenspaces of $S$. Here we are using again that $f$ is substantial. This splitting trivially satisfies condition (2). Since the $\alpha$-splitting is maximal, we conclude that $\Lambda_{k}^{+}=0$ or $\Lambda_{k}^{-}=0$. Therefore, $B_{k}^{+}=0$ or $B_{k}^{-}=0$.

Let $\Gamma_{i}$ be circular and $N_{i} \subset \Lambda_{k}, i \geqslant 1$. We call $\Gamma_{i}$ pseudoholomorphic if the complex structure $J \in K_{i}$ for $\Gamma_{i}$ extends to a parallel complex structure $J \in L$ on $\Lambda_{k}$ along $f$. This means all leaves of $\Gamma_{i}$ are pseudoholomorphic; cf. Lemma 4.3.

Lemma 4.6. Let $\Gamma_{i}$ be circular with complex structure $J$. If $T \in H$ and $T \mid \Gamma_{i}=J_{\theta}=e^{\theta J}, 0<\theta<\pi$, then $\Gamma_{i}$ is pseudoholomorphic.

Proof. Let us first assume $T \mid \Gamma_{i}=J$. Then we have $A_{S^{2} \xi}=A_{\xi} J^{2}=-A_{\xi}$ on $\Gamma_{i}$, so $A_{\left(S^{2}+I\right) \xi}=0$. This implies as before that $S^{2}=-I$ on $\Lambda_{k} \supset N_{i}$.

The general case can be reduced to the above as follows; cf. also Proposition 1.6. Define $T^{\prime} \in \tilde{K}$ by $\sin \theta T^{\prime}=T-\cos \theta I$ on $\Gamma_{i}, T^{\prime}=T$ on $\Gamma_{i}{ }^{\perp}$. Consider $S^{\prime}$ with $\sin \theta S^{\prime}=S-\cos \theta I$ on $\Lambda_{k}, S^{\prime}=S$ on $\Lambda_{k}^{\perp}$. We conclude as in Proposition 1.6 that $S^{\prime}$ is orthogonal. Therefore $T^{\prime} \in H$ and $T^{\prime 2}=-I$. 
Define subgroups of $H$ by $H_{k}=\left\{T \in H \mid T=I\right.$ on $\left.B_{k}^{\perp}\right\}$, and the canonical injection $\delta: H_{1} \times \cdots \times H_{r} \rightarrow H, \delta\left(T_{1}, \cdots, T_{r}\right)=T_{1} \cdots T_{r}$. An easy consequence of Lemmas 4.5 and 4.6 is the following

Proposition 4.7. (i) $H_{k}=S^{1}=\mathbf{R} / 2 \pi \mathbf{Z}$ if $B_{k}$ is pseudoholomorphic, and $H_{k}=\mathbf{Z}_{2}=\{I,-I\}$ otherwise.

(ii) $\delta$ is an isomorphism.

We have now completely proved the main result of this paper. It is convenient to introduce the (reduced) multiplicity $m_{k} \geqslant 0$ of $\Lambda_{k}$, where $m_{k}+1$ is the number of distinct $\Gamma_{i}$ such that $N_{i} \subset \Lambda_{k}$.

Theorem 4.8. Let $f$ be an isometric immersion of a simply connected riemannian manifold $M^{n}$ into euclidean space $\mathbf{R}^{n+p}$.

(i) The associated family $f_{\Re}$ (i.e., all isometric immersions $M^{n} \rightarrow \mathbf{R}^{n+p}$ that have the same Gauss map as $f$, up to congruences) is parametrized by a compact abelian group $\mathfrak{\Omega}$.

(ii) The structure of this group is

$$
\mathfrak{\Omega} \cong \mathbf{Z}_{2}^{m_{1}} \times \cdots \times \mathbf{Z}_{2}^{m_{s}} \times S^{1} \times \cdots \times S^{1},
$$

with one factor $S^{1}=\mathbf{R} / \pi \mathbf{Z}$ for each factor in the A-splitting of the tangent bundle that is circular, but not pseudoholomorphic.

The groups $\mathbf{Z}_{2}^{m_{k}}$ arise from the factors $\Lambda_{k}$ of the $\alpha$-splitting. More precisely, $\mathbf{Z}_{2}^{m_{k}}$ is the quotient group $\mathbf{Z}_{2}^{m_{k}+1}$ modulo the diagonal subgroup $\{I,-I\}$, which is trivial iff $\Lambda_{k}$ contains only one $N_{i}$. Clearly, $0 \leqslant \operatorname{dim} \Omega \leqslant n / 2$ and $0 \leqslant m=m_{1}+\cdots+m_{s} \leqslant \min \{p, n-1\}$.

The discrete part of $\Omega$ is a global phenomenon. In fact, any point $x \in M$ has an open neighborhood $U$ where $f$ is a product corresponding to the $A$-splitting of $f \mid U$, which thus has only simple factors. We now mention a condition for $M$ which implies that $\Re$ cannot have a toral component.

Corollary 4.10. If $M^{n}$ has nonnegative Ricci curvature, then $\Re$ is discrete.

Proof. All (local) circular factors are minimal, and therefore totally geodesic.

Corollary 4.11. Let $f, \tilde{f}: M^{2} \rightarrow \mathbf{R}^{2+p}$ be isometric immersions with the same Gauss map, $M^{2}$ connected.

(i) If $M^{2}$ is oriented and $f$, $\tilde{f}$ have the same oriented Gauss map, then $f$ and $\tilde{f}$ are congruent, unless they are associated minimal surfaces, and not (pseudo)holomorphic.

(ii) If the mean curvature of $f$ is nonzero at some point, then $f$ and $\tilde{f}$ are congruent, except possibly when $M^{2}$ is flat.

The first half of (i) was also proved in [13]; part (ii) is contained in [2] for $p=1$. Notice that in (ii), there can be at most two congruence classes of immersions. 
Proof. It is sufficient to observe that for $n=2$, the group $\Re$ for the universal cover $\tilde{M}^{2} \rightarrow M^{2} \rightarrow \mathbf{R}^{2+p}$ is either trivial, $S^{1}$, or $\mathbf{Z}_{2}$. In the last case, $M^{2}$ is locally a product of curves.

Corollary 4.12. Let $f, \tilde{f}: M^{n} \rightarrow \mathbf{R}^{n+1}$ be isometric immersions with the same Gauss map, $M^{n}$ connected. Then $f$ and $\tilde{f}$ are congruent if any one of the following conditions holds for $f$ :

(i) The rank of the second fundamental form $A$ is $n \geqslant 3$ at some point $x_{0} \in M$.

(ii) There exists $x_{0} \in M$ such that the mean curvature $H\left(x_{0}\right) \neq 0$, and the set $\{x \in M \mid A(x) \neq 0\}$ is connected.

(iii) The immersion $f$ is minimal, unless $f$ is circular (up to a euclidean factor); cf. also Corollary 2.6.

Part (i) and a weak version of (ii) were proved in [2].

Proof. The group $\mathfrak{A}^{\prime}$ for the universal cover $\tilde{M}^{n} \rightarrow M^{n} \rightarrow \mathbf{R}^{n+1}$ is either trivial, $\mathbf{Z}_{2}$, or $S^{1}$.

(i) The $A$-decomposition of $f$ is trivial. Thus $\Re=\mathbf{Z}_{2}$ is impossible. But if $\mathfrak{\Re}=S^{1}$, then $f$ is circular, and rank $A \leqslant 2$ everywhere.

(ii) $\Re=\mathbf{Z}_{2}$, if not trivial. It follows that $T M=\Gamma_{0} \oplus \Gamma_{1} \oplus \Gamma_{2}$, say $A \Gamma_{2}\left(x_{0}\right)$ $\neq 0$. Now $A$ vanishes locally either on $\Gamma_{1}$ or $\Gamma_{2}$, since $f$ splits accordingly because of codimension 1. But $\{x \in M \mid A(x) \neq 0\}$ is connected. Therefore, $A \Gamma_{1}$ and thus $\Gamma_{1}$ is zero everywhere. This is a contradiction.

(iii) Since $f$ is analytic, $T M=\Gamma_{0} \oplus \Gamma_{1}$ globally, so $\Re=S^{1}$, if not trivial, and $\Gamma_{1}$ is circular.

To illustrate how $\mathbf{Z}_{2}$-factors of $\Re$ may arise, we give a typical example. Let $\phi, \psi: \mathbf{R} \rightarrow \mathbf{R}$ be smooth functions, not identically zero, such that $\phi(t)=\psi(t)$ $=0$ for $t \geqslant 0$. For $n \geqslant 2$, consider the region $U=U_{1} \cup U_{2}$ in $\mathbf{R}^{n}$, where $U_{i}=\left\{x \mid x_{i}>0\right\}$. Define real valued functions $\theta_{ \pm}$on $U$ by $\theta_{ \pm}(x)= \pm \phi\left(x_{1}\right)$ on $U_{2}$, and $\theta(x)=\psi\left(x_{2}\right)\left(x_{3}^{2}+\cdots+x_{n}^{2}\right)$ on $U_{1}$. Note that $\theta_{ \pm}=0$ on $U_{1} \cap U_{2}$. Then the graphs $f_{ \pm}: U \rightarrow \mathbf{R}^{n+1}$ of $\theta_{ \pm}$provide examples of noncongruent isometric immersions (in the induced metric), with congruent Gauss maps.

\section{References}

[1] K. Abe, On a class of hypersurfaces of $\mathbf{R}^{2 n+1}$, Duke Math. J. 41 (1974) 865-874.

[2] K. Abe \& J. Erbacher, Isometric immersions with the same Gauss map, Math. Ann. 215 (1975) 197-201.

[3] J. L. Barbosa, On minimal immersions of $S^{2}$ into $S^{2 m}$, Trans. Amer. Math. Soc. 210 (1975) 75-106.

[4] R. Bryant, Conformal and minimal immersions of compact surfaces into the 4-sphere, J. Differential Geometry 17 (1982) 455-473. 
[5] E. Calabi, Isometric imbedding of complex manifolds, Ann. of Math. (2) 58 (1953) 1-23.

[6] Quelques applications de l'analyse complexe aux surfaces d'aire minima, Topics in Complex Manifolds, University of Montreal, Montreal, Canada, 1968, 59-81.

[7] S. S. Chern, On the minimal immersions of the two sphere in a space of constant curvature, Problems in Analysis, Princeton University Press, Princeton, 1970, 27-40.

[8] M. Dajczer \& D. Gromoll, Gauss parametrizations and rigidity aspects of submanifolds, J. Differential Geometry 22 (1985) 1-12.

[9] M. Dajczer \& L. Rodriguez, Rigidity of real Kaehler submanifolds, Preprint, I.M.P.A., Rio de Janeiro, 1985, to appear in Duke Math. J.

[10] N. Ejiri, The index of minimal immersions of $S^{2}$ into $S^{2 n}$, Math. Z. 184 (1983) 172-182.

[11] C. C. Fwu, Kaehler manifolds isometrically immersed in euclidean space, J. Differential Geometry 14 (1979) 99-103.

[12] P. Hartman, On isometric immersions in Euclidean space of manifolds with non-negative sectional curvature. II, Trans. Amer. Math. Soc. 147 (1970) 529-540.

[13] D. Hoffman \& R. Osserman, The Gauss map of surfaces in $\mathbf{R}^{n}$, J. Differential Geometry 18 (1983) 733-754.

[14] H. B. Lawson, Complete minimal surfaces in $S^{3}$, Ann. of Math. (2) 92 (1970) 335-374.

[15] _ Some intrinsic characterizations of minimal surfaces, J. Analyse Math. 29 (1971) $151-161$.

[16] _ Proc. Sympos. Pure Math., Vol. 27, Amer. Math. Soc., Providence, RI, 1975.

[17] Lectures on minimal submanifolds. I, Publish or Perish, Boston, 1980.

[18] J. D. Moore, Isometric immersions of Riemannian products, J. Differential Geometry 5 (1971) 159-168.

[19] P. Ryan, Kaehlerian manifolds as real hypersurfaces, Duke Math. J. 40 (1973) 207-214.

[20] T. Takahashi, A note on Kaehlerian hypersurfaces of spaces of constant curvature, Kumamoto J. Sci. (Math.) 9 (1972) 21-24.

I.M.P.A., Rio DE JANEIRO, BRAZIL

State University of New York, Stony Brook 